\title{
Identification of a Syndrome Class of Neuropsychiatric Adverse Reactions to Mefloquine from Latent Class Modeling of FDA Adverse Event Reporting System Data
}

\author{
Remington L. Nevin ${ }^{1}$ Jeannie-Marie Leoutsakos ${ }^{2,3}$
}

Published online: 6 January 2017

(c) The Author(s) 2017. This article is published with open access at Springerlink.com

\begin{abstract}
Introduction Although mefloquine use is known to be associated with a risk of severe neuropsychiatric adverse reactions that are often preceded by prodromal symptoms, specific combinations of neurologic or psychiatric reactions associated with mefloquine use are not well described in the literature. This study sought to identify a distinct neuropsychiatric syndrome class associated with mefloquine use in reports of adverse events.

Methods Latent class modeling of US Food and Drug Administration Adverse Event Reporting System (FAERS) data was performed using indicators defined by the Medical Dictionary for Regulatory Activities neurologic and psychiatric high-level group terms, in a study dataset of FAERS reports $(n=5332)$ of reactions to common antimalarial drugs.

Results A distinct neuropsychiatric syndrome class was identified that was strongly and significantly associated with reports of mefloquine use (odds ratio $=3.92,95 \%$ confidence interval 2.91-5.28), defined by a very high probability of symptoms of deliria $(82.7 \%)$ including confusion and disorientation, and a moderate probability of other severe psychiatric and neurologic symptoms including dementia and amnesia (18.6\%) and seizures (18.1\%).
\end{abstract}

Remington L. Nevin

rnevin@jhu.edu

1 Department of Environmental Health and Engineering, Johns Hopkins Bloomberg School of Public Health, 615 N. Wolfe St., Baltimore, MD 21205, USA

2 Department of Psychiatry and Behavioral Sciences, Johns Hopkins School of Medicine, Baltimore, MD, USA

3 Department of Mental Health, Johns Hopkins Bloomberg School of Public Health, Baltimore, MD, USA
The syndrome class was also associated with symptoms that are considered prodromal including anxiety, depression, sleep disturbance, and abnormal dreams, and neurological symptoms such as dizziness, vertigo, and paresthesias.

Conclusions This study confirms in FAERS reports the existence of a severe mefloquine neuropsychiatric syndrome class associated with common symptoms that may be considered prodromal. Clinical identification of the characteristic symptoms of this syndrome class may aid in improving case finding in pharmacovigilance studies of more serious adverse reactions to the drug.

\section{Key Points}

Some reports of adverse effects from mefloquine define a syndrome class characterized by symptoms of deliria.

This syndrome class is associated with additional symptoms including some that may be prodromal.

\section{Introduction}

Use of mefloquine, a synthetic quinoline derivative antimalarial, has been well known for many decades to be associated with a risk of occasionally severe, neuropsychiatric adverse reactions [1]. When used prophylactically to prevent malaria, certain of these reactions may be considered prodromal, and as described in a recent boxed warning mandated by the US Food and Drug 
Administration (FDA) in 2013, may predict the development of more serious adverse effects, including potentially disabling psychiatric and neurological symptoms that may persist or become permanent after mefloquine has been discontinued $[2,3]$.

Symptoms consistent with prodromal reactions to mefloquine, which include very common symptoms of sleep disturbance and abnormal dreams, which are reported in greater than $10 \%$ of users, and common symptoms of anxiety and depression, which are reported in between 1 and $10 \%$ of users [2], may occur independently in settings such as international travel [4] and military operations [5] where mefloquine may be used prophylactically. Such prodromal reactions may also be plausibly reported as a result of reporting bias, or because of nocebo effects [6]. Certain symptoms consistent with those of the mefloquine prodrome may also occur from other drugs in settings of mefloquine use. For example, two other antimalarial drugs commonly used for prophylaxis of chloroquine-resistant malaria, atovaquone-proguanil and doxycycline, are also associated with symptoms of sleep disturbance and abnormal dreams [7], although these are reported less commonly than with mefloquine [8-10]. Similarly, chloroquine has also long been associated with symptoms similar to those caused by mefloquine [11, 12], although as with atovaquone-proguanil and doxycycline, their occurrence with the use of chloroquine is not accompanied by warnings to discontinue the drug at their onset.

Owing to the perceived ubiquity of such symptoms in settings of mefloquine use, the unique guidance applicable to mefloquine to discontinue the drug at the onset of prodromal symptoms such as sleep disturbance, abnormal dreams, anxiety, and depression may risk being minimized or overlooked [2]. Similarly, as demonstrated by case reports [13-15], such symptoms may be misattributed by the patient or the clinician to causes other than to mefloquine. In each case, this may potentially reduce the effectiveness of regulatory guidance that the drug be discontinued at their onset [2].

While US regulators at the FDA have noted that more serious neurologic reactions to mefloquine are typically accompanied by lasting psychiatric symptoms [3, 16], specific combinations of neurologic and psychiatric reactions associated with mefloquine use are not well described in the literature. The absence in the literature of a wellcharacterized and specific syndrome of neuropsychiatric adverse reactions associated with mefloquine use may risk such combinations of reactions confounding the diagnosis of numerous other neuropsychiatric disorders [17, 18], which may consequently limit the specificity of case finding in pharmacovigilance studies of adverse reactions.

This study therefore sought to identify a distinct neuropsychiatric syndrome class associated with reports of adverse reactions from mefloquine use. This study further sought to confirm the association of this syndrome with prodromal symptoms, and to identify other specific symptoms commonly associated with it that might inform improvements in case finding.

\section{Methods}

Latent class modeling (LCM) is a statistical method enabling the grouping of individuals into one or more distinct classes on the basis of responses to a finite number of indicators, such as the presence or absence of various reported symptoms [19]. LCM is a commonly accepted method for improving accuracy in psychiatric diagnosis in the absence of an objective "gold standard" [20]. Although the reporting of drug adverse reactions may involve selecting from potentially thousands of distinct medical terms, these may be categorized into a manageable number by means of the Medical Dictionary for Regulatory Activities (MedDRA) vocabulary, which translates reported reactions into standard terminology known as lowest level terms, and groups these into medically similar or equivalent preferred terms (PTs) [21]. The MedDRA further categorizes PTs multiaxially into one or more of 26 top-level system organ classes (SOCs), and within each SOC, to typically one of a few dozen distinct high-level group terms (HLGTs) [21], thus making the presence or absence of reactions categorized at the SOC or HLGT level of potential utility as indicators in LCM.

In this analysis, both SOC- and HLGT-level indicators were used in conducting case-level LCM among drug adverse events reported to the FDA. Reports used in this analysis included those for the three antimalarial drugs commonly used in the prophylaxis of chloroquine-resistant malaria, mefloquine, atovaquone-proguanil, and doxycycline; and as controls, those for chloroquine and the antimotility agent loperamide, a common co-exposure in settings where antimalarials are used [22].

Descriptive statistical analyses were conducted using STATA Version 14 (StataCorp, College Station, TX, USA), and LCM was conducted using MPLUS Version 7.4 (Muthen \& Muthen, Los Angeles, CA, USA).

\subsection{Study Data}

Reports of adverse reactions are maintained by the FDA in the FDA Adverse Event Reporting System (FAERS) [23]. Study data were obtained by querying the Qscan FDA database (DrugLogic, Reston, VA, USA), a commercial aggregator of publicly available FAERS data, for all FAERS reports listing mefloquine, atovaquone-proguanil, doxycycline, chloroquine, and loperamide or their common 
generic and trade variants as suspect or non-suspect drugs through 2012, the year prior to the FDA boxed warning. Duplicate reports based on same age, sex, and FDA receipt dates were automatically excluded from the query.

\subsection{MedDRA Categorization of FAERS Report Reactions}

Where reactions in historical FAERS reports did not match a current MedDRA PT, these were updated to the current MedDRA vocabulary if these matched a current lowest level term, or where there was no match, manually to a lexical variant of a current lowest level term. Where a PT was multiaxial, PT assignment was prioritized to the SOC psychiatric disorders and nervous system disorders, in that order. Categorized reactions of a general, social, or procedural nature (i.e., SOCs general disorders and administration-site conditions; investigations; injury, poisonings, and procedural complications; surgical and medical procedures; and social circumstances) were excluded. Reactions describing congenital and perinatal conditions (i.e., SOCs congenital, familial, and genetic disorders; and pregnancy, puerperium, and perinatal conditions), neoplastic conditions (i.e., SOC neoplasms benign, malignant and unspecified including cysts and polyps), immune and infectious disorders (i.e., SOCs immune system disorders; and infections and infestations), and conditions of the reproductive system (i.e., SOC reproductive system and breast disorders) were also excluded.

For each FAERS report, binary indicators corresponding to the 15 remaining SOCs, and to individual HLGTs within the psychiatric and nervous system disorder SOCs, were created on the basis of reported PTs.

\subsection{Study Datasets}

By drug, datasets were created collapsing by case number any positive response in binary SOC- and HLGT-level indicators across original and subsequent FAERS reports. By case, the minimum of reported age (when not unknown) was assigned as case age; sex was assigned as male, female, or unknown; and for the three primary study drugs mefloquine, atovaquone-proguanil, and doxycycline, the indication was assigned as either for malaria prophylaxis or other/unknown. To exclude neonatal cases potentially due to maternal exposure, cases less than 1 year of age were excluded. Datasets by drug were then pooled, and to ensure independence in subsequent LCM, cases appearing in multiple study drug datasets were excluded. Two study datasets were created from the pooled dataset: the primary dataset consisting of mefloquine, atovaquone-proguanil, and doxycycline cases; and a control dataset consisting of chloroquine and loperamide cases.

\subsection{Latent Class Indicator Selection}

To permit the data-driven selection of specific indicators for the final LCM, initial latent class models were first estimated from SOC-level indicators in the primary dataset with greater than $2 \%$ overall prevalence with the aim of identifying broad neuropsychiatric classes of reaction from which specific neuropsychiatric HLGT-level indicators would then be selected.

Two separate models were first estimated to assess for convergent results across models: the first, a fully conditionally dependent (full) model in which conditional dependence was permitted between all indicator pairs; and second, a partially conditionally dependent (partial) model in which conditional dependence was permitted between selected indicator pairs. For the partial model, these were iteratively fit, specifying conditional dependence between SOC-level indicator pairs with significant pairwise bivariate residuals (Pearson $\chi^{2}>1.96$ ), until a final model could be estimated with non-significant pairwise bivariate residuals. Among each model, fit statistics were compared, with the final model selection made based on Bayesian information criteria (BIC) [19]. The partial model was evaluated for evidence of residual conditional dependence by means of multiple, random probability-weighted pseudoclass assignment, whereby SOC-level indicators with Bonferroni-adjusted significant $p$ values from pairwise logistic regression in five or more of 100 draws by pseudoclass were deemed to exhibit significant conditional dependence.

From each selected model, multiple neuropsychiatric classes were then identified based on relatively elevated conditional probabilities for psychiatric and nervous system disorder SOC-level indicators. Among cases with the most-likely membership in the identified neuropsychiatric classes, neuropsychiatric HLGT-level indicators were then similarly evaluated for evidence of differential measurement by drug by means of multiple, random probabilityweighted pseudoclass assignment [24]. HLGT-level indicators free of evidence of significant differential measurement across model neuropsychiatric classes, and with greater than $2 \%$ overall prevalence, were considered for selection for the final LCM.

\subsection{HLGT-Level LCM}

Latent class models with two through four classes were estimated from the primary dataset from the selected neuropsychiatric HLGT-level indicators, permitting pairwise conditional dependence between all indicators, with the final model selection made based on BIC. To account for uncertainty in class membership, association of HLGTlevel class with drug was assessed by corrected three-step testing in MPLUS, per published methods [25] with results 
reported as odds ratios (ORs) and $95 \%$ confidence intervals (95\% CIs).

\section{Results}

The number of cases by drug and final dataset are shown in Fig. 1. When pooled, 196 of the 12,674 cases (1.5\%) were excluded for listing multiple study drugs. The sex and age group of study cases by drug are shown in Table 1 . The age and sex distribution of cases varied significantly by $\chi^{2}$ test by drug for age group $(p<0.001)$ and for $\operatorname{sex}(p<0.001)$ in both the primary dataset and the pooled data overall. In the primary dataset, the distribution of reported indication also varied significantly by $\chi^{2}$ test by drug $(p<0.001)$, as shown in Table 2.

\subsection{SOC-Level Modeling}

Total numbers and proportions of cases with reported reactions in each of the 15 study SOCs were tabulated by drug in the primary dataset and are shown in Table 3. The proportions of reported reactions varied significantly by $\chi^{2}$ test by drug across all SOCs (all $p<0.05$ ). By drug, the most commonly reported reactions were within the psychiatric disorders SOC for mefloquine (56.5\% of cases, most commonly the PT anxiety), the nervous system disorders SOC for atovaquone-proguanil (29.1\% of cases, most commonly the PT headache), and the gastrointestinal disorders SOC for doxycycline $(31.4 \%$ of cases, most commonly the PT nausea). Reactions were reported in fewer than $2 \%$ of cases overall within two SOCs, ear and labyrinth disorders, and endocrine disorders, which were excluded from subsequent LCM.

In the primary dataset, reactions within the nervous system disorder SOC were reported for 20 HLGTs at frequencies ranging from 0.03 to $22.2 \%$, and within the psychiatric disorder SOC for 23 HLGTs at frequencies ranging from 1.1 to $12.6 \%$.

In the SOC-level LCM, full models with two through five classes had BIC of 54,213, 54,162, 54,158, and 54,193; and partial models with two through seven classes had BIC of 54,006, 53,994, 53,967, 53,965, 53,937, and 53,940, respectively. The four-class full and six-class partial models were selected based on the best fit by BIC. Class labels were assigned subjectively based on conditional probabilities, with a mild and moderate neuropsychiatric class identified in each, as shown in Fig. 2.

The partial model had entropy of 0.560 and a non-significant overall univariate residual $\left(\chi^{2}=0.003, p>0.05\right)$. Despite non-significant pairwise bivariate residuals $\left(\chi^{2}<\right.$ 1.96, $p>0.05$ for all comparisons), the overall bivariate residual was significant $\left(\chi^{2}=13.79, p>0.05\right)$, with evidence on subsequent pseudoclass testing of significant residual conditional dependence between indicator pairs for the respiratory and vascular SOCs, and for the hepatobiliary and metabolic SOCs in the moderate neuropsychiatric class; and between indicator pairs for the cardiac and renal SOCs in the multi-system class. In contrast, the full model had entropy of 0.872 , and non-significant
Fig. 1 Study cases in the primary and control datasets. $A P$ atovaquone-proguanil, $C Q$ chloroquine, $D X$ doxycycline, $L P$ loperamide, $M Q$ mefloquine

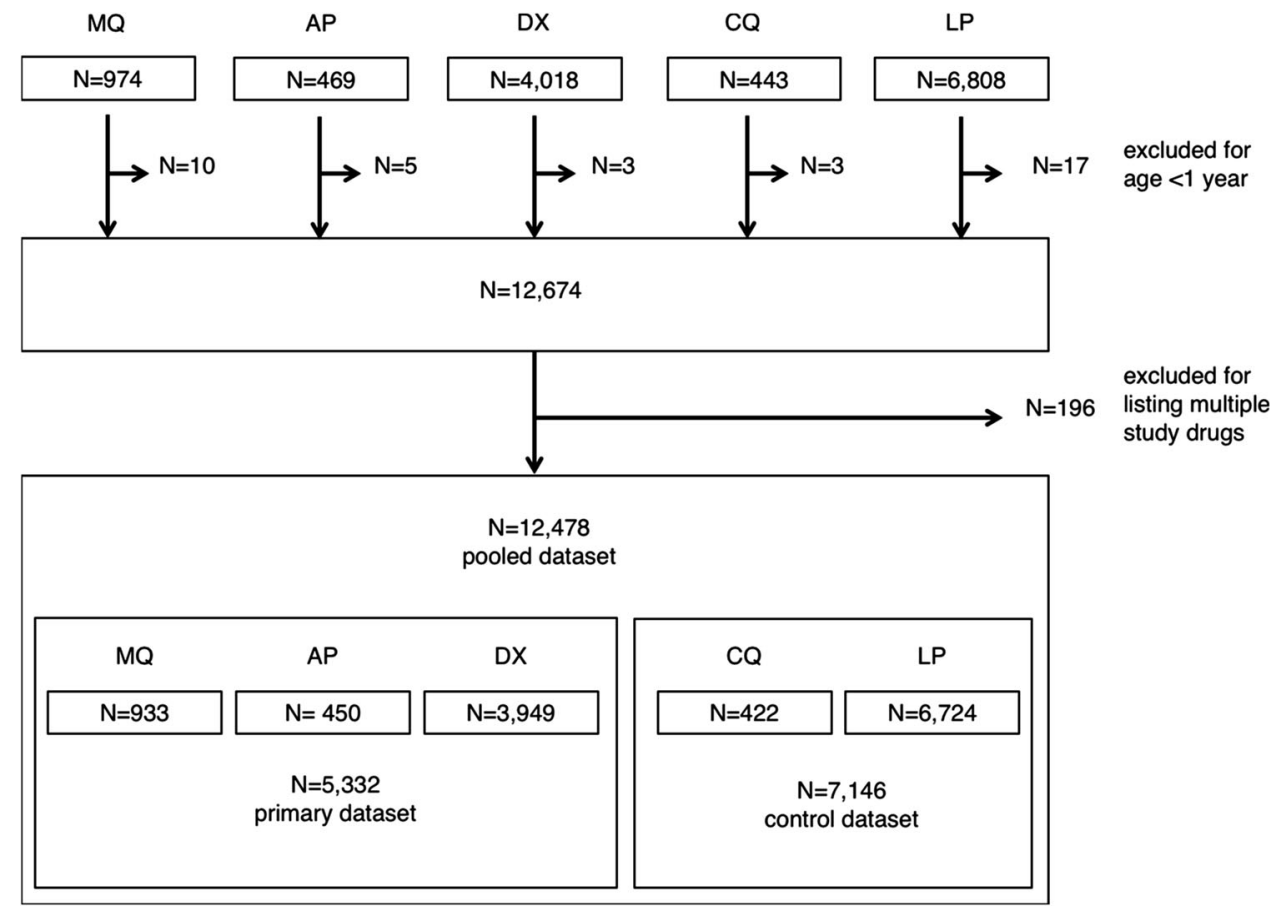


Table 1 Sex and age groups of study cases in the primary and control datasets, by drug

\begin{tabular}{|c|c|c|c|c|c|}
\hline & \multicolumn{3}{|c|}{ Primary dataset } & \multicolumn{2}{|c|}{ Control dataset } \\
\hline & $\begin{array}{l}\text { Mefloquine } \\
n(\%)\end{array}$ & $\begin{array}{l}\text { Atovaquine-proguanil } \\
n(\%)\end{array}$ & $\begin{array}{l}\text { Doxycycline } \\
n(\%)\end{array}$ & $\begin{array}{l}\text { Chloroquine } \\
n(\%)\end{array}$ & $\begin{array}{l}\text { Loperamide } \\
n(\%)\end{array}$ \\
\hline All cases & $933(100)$ & $450(100)$ & 3949 (100) & $422(100)$ & $6724(100)$ \\
\hline \multicolumn{6}{|l|}{ Sex } \\
\hline Male & 489 (52.4) & $198(44.0)$ & $1506(38.1)$ & 109 (25.8) & 3306 (49.2) \\
\hline Female & 418 (44.8) & $224(49.8)$ & 2308 (58.5) & $300(71.1)$ & 3194 (47.5) \\
\hline Unknown & $26(2.8)$ & $28(6.2)$ & $135(3.4)$ & $13(3.1)$ & $224(3.3)$ \\
\hline \multicolumn{6}{|c|}{ Age group, years } \\
\hline$<18$ & $51(5.5)$ & $35(7.8)$ & $192(4.9)$ & $22(5.2)$ & $181(2.7)$ \\
\hline $18-65$ & $595(63.8)$ & $225(50)$ & 1917 (48.5) & $216(51.2)$ & 2659 (39.5) \\
\hline $65+$ & $287(30.8)$ & $190(42.2)$ & 1840 (46.6) & 184 (43.6) & $3884(57.8)$ \\
\hline
\end{tabular}

Across all strata, proportions varied significantly by drug, in both the primary dataset and in the pooled data overall $\left(p<0.05\right.$ by $\chi^{2}$ test $)$

\begin{tabular}{lllr}
\hline & \multicolumn{2}{l}{ Primary dataset } & \\
\cline { 2 - 4 } & $\begin{array}{l}\text { Mefloquine } \\
n(\%)\end{array}$ & $\begin{array}{l}\text { Atovaquone-proguanil } \\
n(\%)\end{array}$ & $\begin{array}{l}\text { Doxycycline } \\
n(\%)\end{array}$ \\
\hline $\begin{array}{l}\text { All cases } \\
\text { Drug indication }\end{array}$ & $933(100)$ & $450(100)$ & $3949(100)$ \\
$\begin{array}{l}\text { Malaria prophylaxis } \\
\text { Other or unknown }\end{array}$ & $294(31.5)$ & $278(61.8)$ & $30(0.8)$ \\
\hline
\end{tabular}

Proportions varied significantly by drug $\left(p<0.001\right.$ by $\chi^{2}$ test $)$
Table 2 Drug indication of study cases in the primary dataset, by drug amnestic conditions; depressed mood disorders and disturbances; neuromuscular disorders; peripheral neuropathies; psychiatric disorders NEC; and seizures (including subtypes).

In the HLGT-level LCM, models with two through four classes had BIC of 11,908, 11,972, and 12,038, respectively. The two-class model was selected, and had entropy of 0.971 , and non-significant overall univariate and bivariate as well as pairwise bivariate residuals. A neuropsychiatric syndrome class with an overall prevalence of $4.3 \%$ was identified without evidence of significant differential measurement by drug for any HLGTlevel indicator. In contrast, the non-syndrome class demonstrated evidence of significant differential measurement for three HLGT-level indicators: deliria (including confusion), dementia and amnestic conditions, and depressed mood disorders and disturbances. Among cases in the most-likely non-syndrome class, simple logistic regression showed these indicators were significantly more likely to be reported with mefloquine, and significantly less likely to be reported with doxycycline. Conditional probabilities of HLGT-level indicators for the syndrome and non-syndrome class are reported in Table 5. 
Table 3 Prevalence of reported reactions by MedDRA SOC, by drug and overall, primary dataset

\begin{tabular}{|c|c|c|c|c|}
\hline & $\begin{array}{l}\text { Mefloquine } \\
n(\%)\end{array}$ & $\begin{array}{l}\text { Atovaquone-proguanil } \\
n(\%)\end{array}$ & $\begin{array}{l}\text { Doxycycline } \\
n(\%)\end{array}$ & $\begin{array}{l}\text { Overall } \\
n(\%)\end{array}$ \\
\hline Any SOC & $933(100)$ & $450(100)$ & $3949(100)$ & $5332(100)$ \\
\hline Blood and lymphatic system disorders & $55(5.6)$ & $42(9.3)$ & $487(12.3)$ & $584(11.0)$ \\
\hline Cardiac disorders & $136(14.6)$ & $19(4.2)$ & $473(12.0)$ & $628(11.8)$ \\
\hline Ear and labyrinth disorders ${ }^{\mathrm{a}}$ & $33(3.5)$ & $6(1.3)$ & $70(1.8)$ & $109(2.0)$ \\
\hline Endocrine disorders $^{\mathrm{a}}$ & $7(0.8)$ & $2(0.4)$ & $70(1.8)$ & $79(1.5)$ \\
\hline Eye disorders & $67(7.2)$ & $25(5.6)$ & $212(5.4)$ & $304(5.7)$ \\
\hline Gastrointestinal disorders & $233(25.0)$ & $123(27.3)$ & $1242(31.4)$ & $1598(30.0)$ \\
\hline Hepatobiliary disorders & $36(3.9)$ & $26(5.8)$ & $326(8.3)$ & $388(7.3)$ \\
\hline Metabolism and nutrition disorders & $67(7.2)$ & $33(7.3)$ & $447(11.3)$ & $547(10.3)$ \\
\hline Musculoskeletal and connective tissue disorders & $117(12.5)$ & $33(7.3)$ & $614(15.6)$ & $764(14.3)$ \\
\hline Nervous system disorders & $438(47.0)$ & $131(29.1)$ & $1193(30.2)$ & $1762(33.0)$ \\
\hline Psychiatric disorders & $527(56.5)$ & $95(21.1)$ & $805(20.4)$ & $1427(26.8)$ \\
\hline Renal and urinary disorders & $41(4.4)$ & $20(4.4)$ & $381(9.6)$ & $442(8.3)$ \\
\hline Respiratory, thoracic, and mediastinal disorders & $127(13.6)$ & $45(10.0)$ & $767(19.4)$ & $939(17.6)$ \\
\hline Skin and subcutaneous tissue disorders & $148(15.9)$ & $106(23.6)$ & $790(20.0)$ & $1044(19.6)$ \\
\hline Vascular disorders & $66(7.1)$ & $26(5.8)$ & $618(15.6)$ & $710(13.3)$ \\
\hline
\end{tabular}

MedDRA Medical Dictionary for Regulatory Activities, SOC system organ class

Across all SOCs, proportions varied significantly by drug $\left(p<0.05\right.$ by $\chi^{2}$ test)

${ }^{a}$ SOC with $2.0 \%$ prevalence or less overall excluded from subsequent SOC-level analysis

Based on the most-likely class assignment, the prevalence of the syndrome class in the primary study dataset was $10.3 \%$ with mefloquine, $2.0 \%$ with atovaquone-proguanil, and $3.5 \%$ with doxycycline. On three-step testing, the syndrome class was positively associated with mefloquine (OR 3.92, 95\% CI 2.91-5.28, $p<0.001$ ), and negatively associated with atovaquone-proguanil (OR 0.35, 95\% CI $0.14-0.85, p=0.020$ ) and doxycycline (OR 0.38 , 95\% CI 0.28-.51, $p<0.001)$. Applying the fitted model to the control dataset, based on the most-likely class assignment, the prevalence of the syndrome was $3.3 \%$ with chloroquine and $6.0 \%$ with loperamide. However, on threestep testing, there was no significant association of the syndrome with chloroquine $(\mathrm{OR}=0.46,95 \%$ CI $0.17-1.28$, $p=0.138)$ or with loperamide $(\mathrm{OR}=2.172,95 \% \mathrm{CI}$ $0.78-6.04, p=0.138)$.

\subsection{Most-Likely Class Modeling}

On post-hoc analysis of the study dataset, assigning cases to their most-likely class, among the 5095 cases assigned to the non-syndrome class, the five most commonly reported HLGTs across all 15 study SOCs were gastrointestinal signs and symptoms; neurological disorders NEC; epidermal and dermal conditions; respiratory disorders NEC; and anxiety disorders and symptoms. In contrast, among the 237 cases assigned to the most-likely syndrome class, the five most commonly reported HLGTs were deliria (including confusion); neurological disorders NEC; anxiety disorders and symptoms; sleep disorders and disturbances; and depressed mood disorders and disturbances. The prevalence of these most commonly reported syndrome HLGTs together with the most commonly reported reactions, by PT, within each (with greater than $5 \%$ overall prevalence), by most-likely class, are listed in Table 6, along with ORs for reporting each HLGT in the most-likely syndrome vs. non-syndrome class.

Among the study dataset, among cases assigned to the most-likely class, the proportion of cases by drug in the syndrome class did not vary significantly by $\chi^{2}$ test by age group (<18 years, 18-65 years, and 65+ years) for mefloquine $(p=0.292)$, atovaquone-proguanil $(p=0.073)$, or doxycycline $(p=0.151)$. In contrast, while the proportion of cases in the syndrome class by drug did not vary significantly by $\chi^{2}$ test by sex for atovaquone-proguanil ( $p=0.184)$, they did vary significantly by sex for mefloquine $(p=0.001)$ and doxycycline $(p=0.002)$, being strongly associated in each with male sex by simple logistic regression (OR 2.15, 95\% CI 1.37-3.38, and OR 1.70, 95\% CI 1.20-2.41, respectively).

The proportion of cases by drug in the syndrome class also varied significantly by $\chi^{2}$ test by reported indication for mefloquine ( $p<0.001)$, being strongly associated with malaria prophylaxis (OR 2.29, 95\% CI 1.50-3.51) by the most-likely syndrome class assignment, an association not seen with atovaquone-proguanil $(p=0.318)$ or, by 

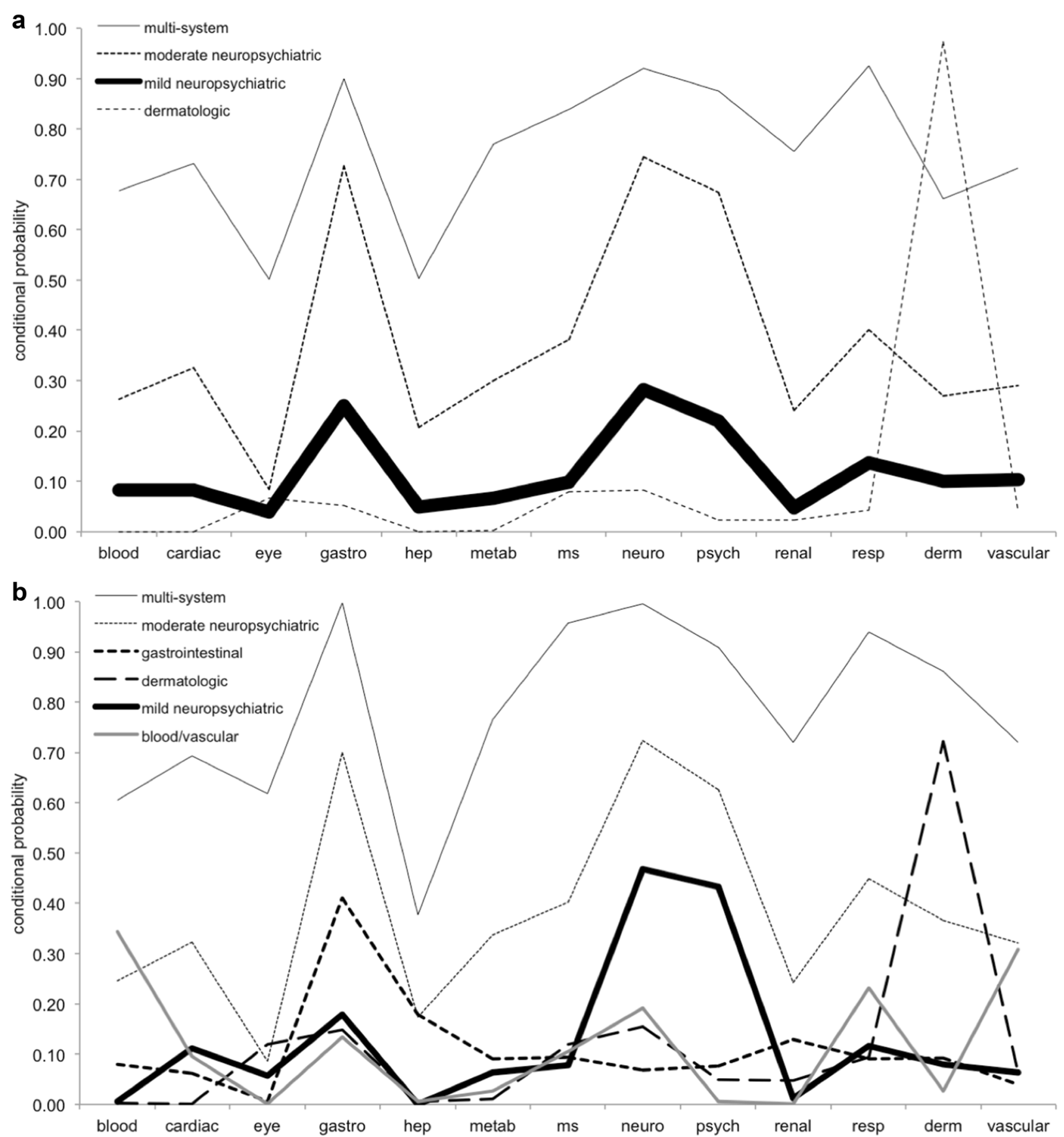

Fig. 2 Conditional probabilities, SOC-level models. a Fully conditionally dependent (full) model. b Partially conditionally dependent (partial) model. blood blood and lymphatic system disorders, cardiac cardiac disorders, derm skin and subcutaneous tissue disorders, eye eye disorders, gastro gastrointestinal disorders, hep hepatobiliary disorders, metab metabolism and nutrition disorders, $m s$

Fischer's exact test, with doxycycline $(p=0.426)$. Overall, based on the most-likely class assignment, the prevalence of the syndrome class in the primary study dataset among musculoskeletal and connective tissue disorders, neuro nervous system disorders, psych psychiatric disorders, renal renal and urinary disorders, resp respiratory, thoracic, and mediastinal disorders, vascular vascular disorders. Line thickness is proportional to class membership conditional probability. SOC system organ class

cases with a reported indication of malaria prophylaxis was $16.0 \%$ with mefloquine, $2.5 \%$ with atovaquone-proguanil, and $0 \%$ with doxycycline. 
Table 4 Prevalence of reported reactions by MedDRA neuropsychiatric HLGT, by SOC-level latent class model most-likely neuropsychiatric class, and overall

\begin{tabular}{|c|c|c|c|c|c|}
\hline & \multicolumn{2}{|l|}{ Moderate } & \multicolumn{2}{|l|}{ Mild } & \multirow{2}{*}{$\begin{array}{l}\text { Overall } \\
n(\%)\end{array}$} \\
\hline & $\begin{array}{l}\text { Partial } \\
n(\%)\end{array}$ & $\begin{array}{l}\text { Full } \\
n(\%)\end{array}$ & $\begin{array}{l}\text { Partial } \\
n(\%)\end{array}$ & $\begin{array}{l}\text { Full } \\
n(\%)\end{array}$ & \\
\hline Any neuropsychiatric HLGT & $511(93.4)$ & $465(92.0)$ & $1577(89.6)$ & $1878(43.0)$ & $2429(45.6)$ \\
\hline Adjustment disorders (including subtypes) & $2^{\mathrm{a}}(0.4)$ & $2^{\mathrm{a}}(0.43)$ & $4^{\mathrm{a}}(0.2)$ & $3^{\mathrm{a}}(0.1)$ & $11(0.21)$ \\
\hline Anxiety disorders and symptoms & $145^{\mathrm{a}}(28.4)$ & $149^{\mathrm{a}}(32.0)$ & $369^{\mathrm{a}}(20.1)$ & $379^{\mathrm{a}}(8.7)$ & $604(11.3)$ \\
\hline Changes in physical activity & $15^{\mathrm{a}}(2.9)$ & $18^{\mathrm{a}}(3.9)$ & $40^{\mathrm{a}}(2.3)$ & $38^{\mathrm{a}}(0.9)$ & $67(1.3)$ \\
\hline CNS infections & $11(2.2)$ & $9(1.9)$ & $18(1.0)$ & $23(0.5)$ & $39(0.7)$ \\
\hline CNS vascular disorders & $29(5.7)$ & $24^{\mathrm{a}}(5.2)$ & $68(3.9)$ & $81(1.9)$ & $134(2.5)$ \\
\hline Cognitive and attention disorders and disturbances & $45^{\mathrm{a}}(8.8)$ & $46^{\mathrm{a}}(9.9)$ & $103^{\mathrm{a}}(5.8)$ & $102^{\mathrm{a}}(2.3)$ & $171(3.2)$ \\
\hline Communication disorders and disturbances ${ }^{\mathrm{b}}$ & $31(6.1)$ & $34(7.3)$ & $75(4.3)$ & $73(1.7)$ & $126(2.4)$ \\
\hline Congenital neurological disorders & $3(0.6)$ & $3(0.6)$ & $3(0.2)$ & $3(0.1)$ & $8(0.2)$ \\
\hline Cranial nerve disorders (excluding neoplasms) & $35^{\mathrm{a}}(6.8)$ & $35^{\mathrm{a}}(7.5)$ & $99^{\mathrm{a}}(5.6)$ & $109^{\mathrm{a}}(2.5)$ & $167(3.1)$ \\
\hline Deliria (including confusion) ${ }^{b}$ & $60(11.7)$ & $55(11.8)$ & $128^{\mathrm{a}}(7.3)$ & $135^{\mathrm{a}}(3.1)$ & $219(4.1)$ \\
\hline Dementia and amnestic conditions ${ }^{\mathrm{b}}$ & $37(7.2)$ & $42(9.0)$ & $92^{\mathrm{a}}(5.2)$ & $92^{\mathrm{a}}(2.1)$ & $161(3.0)$ \\
\hline Demyelinating disorders & $5(1.0)$ & $2(0.4)$ & $19(1.1)$ & $26(0.6)$ & $32(0.6)$ \\
\hline Depressed mood disorders and disturbances ${ }^{\mathrm{b}}$ & $89(17.4)$ & $85(18.3)$ & $184^{\mathrm{a}}(10.4)$ & $192^{\mathrm{a}}(4.4)$ & $340(6.4)$ \\
\hline Developmental disorders NEC & $0(0.0)$ & $0(0.0)$ & $1(0.1)$ & $1(0.0)$ & $1(0.0)$ \\
\hline Dissociative disorders & $6(1.2)$ & $6(1.3)$ & $16^{\mathrm{a}}(0.9)$ & $16^{\mathrm{a}}(0.4)$ & $25(0.5)$ \\
\hline Disturbances in thinking and perception & $41^{\mathrm{a}}(8.0)$ & $43^{\mathrm{a}}(9.2)$ & $176^{\mathrm{a}}(10.0)$ & $179^{\mathrm{a}}(4.1)$ & $242(4.5)$ \\
\hline Eating disorders and disturbances & $29(5.7)$ & $25(5.4)$ & $31(1.8)$ & $36(0.8)$ & $73(1.4)$ \\
\hline Encephalopathies & $15(2.9)$ & $16(3.4)$ & $16(0.9)$ & $20(0.5)$ & $43(0.8)$ \\
\hline Headaches & $131^{\mathrm{a}}(25.6)$ & $121^{\mathrm{a}}(26.0)$ & $190(10.8)$ & $242^{\mathrm{a}}(5.6)$ & $415(7.8)$ \\
\hline Impulse control disorders NEC & $2(0.4)$ & $2(0.4)$ & $12(0.7)$ & $12(0.3)$ & $16(0.3)$ \\
\hline Increased intracranial pressure & $11(2.2)$ & $9(1.9)$ & $50(2.8)$ & $54(1.2)$ & $70(1.3)$ \\
\hline Manic and bipolar mood disorders & $7(1.4)$ & $10(2.2)$ & $40^{\mathrm{a}}(2.3)$ & $40^{\mathrm{a}}(0.9)$ & $55(1.0)$ \\
\hline Mood disorders and disturbances NEC & $73^{\mathrm{a}}(14.3)$ & $74^{\mathrm{a}}(15.9)$ & $193^{\mathrm{a}}(11.0)$ & $203^{\mathrm{a}}(4.6)$ & $318(6.0)$ \\
\hline Movement disorders (including parkinsonism) & $61^{\mathrm{a}}(11.9)$ & $62^{\mathrm{a}}(13.3)$ & $127(7.2)$ & $141^{\mathrm{a}}(3.2)$ & $230(4.3)$ \\
\hline Nervous system neoplasms benign & $0(0.0)$ & $1(0.2)$ & $2(0.1)$ & $2(0.0)$ & $4(0.1)$ \\
\hline Nervous system neoplasms malignant & $0(0.0)$ & $0(0.0)$ & $2(0.1)$ & $3(0.1)$ & $6(0.1)$ \\
\hline Neurological disorders of the eye & $47^{\mathrm{a}}(9.2)$ & $39^{\mathrm{a}}(8.4)$ & $112(6.4)$ & $130^{\mathrm{a}}(3.0)$ & $204(3.8)$ \\
\hline Neurological disorders NEC & $268^{\mathrm{a}}(52.4)$ & $253^{\mathrm{a}}(54.4)$ & $582^{\mathrm{a}}(33.0)$ & $736^{\mathrm{a}}(16.9)$ & $1089(20.4)$ \\
\hline Neuromuscular disorders ${ }^{\mathrm{b}}$ & $43(8.4)$ & $40(8.6)$ & $67(3.8)$ & $82^{\mathrm{a}}(1.9)$ & $163(3.1)$ \\
\hline Peripheral neuropathies ${ }^{\mathrm{b}}$ & $30(5.9)$ & $28(6.0)$ & $32(1.8)$ & $44(1.0)$ & $116(2.2)$ \\
\hline Personality disorders and disturbances & $20^{\mathrm{a}}(3.9)$ & $27^{\mathrm{a}}(5.8)$ & $153^{\mathrm{a}}(8.7)$ & $149^{\mathrm{a}}(3.4)$ & $188(3.5)$ \\
\hline Psychiatric and behavioral symptoms & $26^{\mathrm{a}}(5.1)$ & $30(6.4)$ & $77^{\mathrm{a}}(4.4)$ & $76^{\mathrm{a}}(1.7)$ & $119(2.2)$ \\
\hline Psychiatric disorders NEC ${ }^{\mathrm{b}}$ & $47(9.2)$ & $44(9.5)$ & $71(4.0)$ & $74^{\mathrm{a}}(1.7)$ & $148(2.8)$ \\
\hline Schizophrenia and other psychotic disorders & $17^{\mathrm{a}}(3.3)$ & $13^{\mathrm{a}}(2.8)$ & $82^{\mathrm{a}}(4.7)$ & $85^{\mathrm{a}}(2.0)$ & $109(2.0)$ \\
\hline Seizures (including subtypes) ${ }^{\mathrm{b}}$ & $40(7.8)$ & $37(8.0)$ & $136(7.7)$ & $151(3.5)$ & $203(3.8)$ \\
\hline Sexual dysfunctions and disturbances & $13(2.5)$ & $11(2.4)$ & $18(1.0)$ & $19(0.4)$ & $46(0.9)$ \\
\hline Sleep disorders and disturbances & $119^{\mathrm{a}}(23.3)$ & $113^{\mathrm{a}}(24.3)$ & $273^{\mathrm{a}}(15.5)$ & $291^{\mathrm{a}}(6.7)$ & $454(8.5)$ \\
\hline Sleep disturbances (including subtypes) ${ }^{\mathrm{c}}$ & $0(0.0)$ & $0(0.0)$ & $1(0.1)$ & $1(0.0)$ & $1(0.0)$ \\
\hline Somatoform and factitious disorders & $20(3.9)$ & $19(4.1)$ & $16(0.9)$ & $15(0.3)$ & $48(0.9)$ \\
\hline Spinal cord and nerve root disorders & $16(3.1)$ & $13(2.8)$ & $7(0.4)$ & $10(0.2)$ & $57(1.1)$ \\
\hline Structural brain disorders & $8(1.6)$ & $4(0.9)$ & $10(0.6)$ & $12(0.3)$ & $34(0.6)$ \\
\hline
\end{tabular}


Table 4 continued

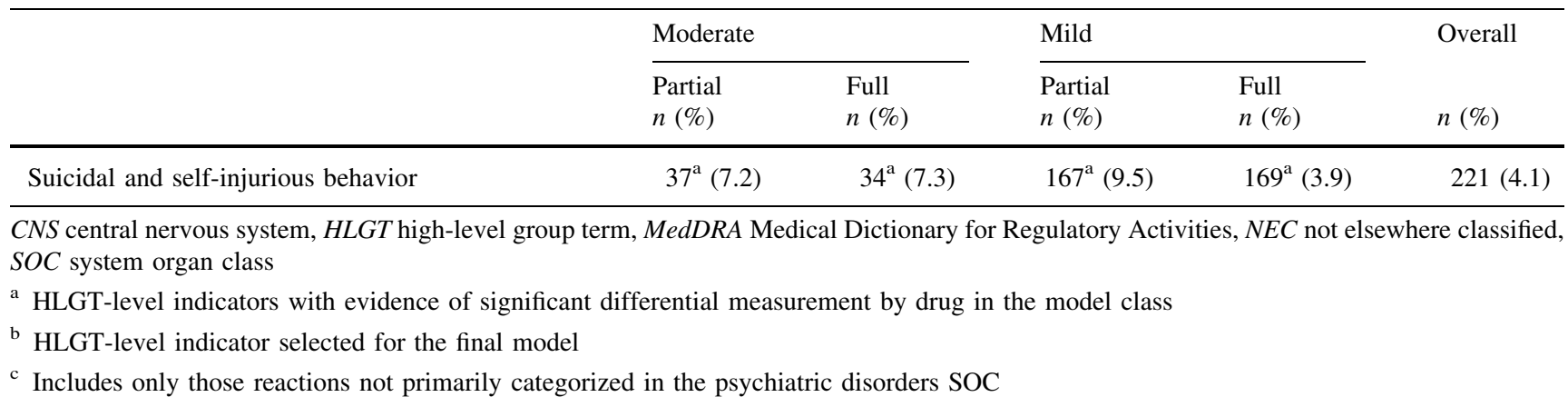

Table 5 Conditional probabilities by class, two-class HLGT-level latent class model

\begin{tabular}{lll}
\hline & Syndrome & Non-syndrome \\
\hline Communication disorders and disturbances & 0.182 & 0.017 \\
Deliria (including confusion) & 0.827 & 0.006 \\
Dementia and amnestic conditions & 0.186 & 0.023 \\
Depressed mood disorders and disturbances & 0.318 & 0.052 \\
Neuromuscular disorders & 0.090 & 0.028 \\
Peripheral neuropathies & 0.082 & 0.019 \\
Psychiatric disorders NEC & 0.314 & 0.015 \\
Seizures (including subtypes) & 0.181 & 0.032 \\
\hline
\end{tabular}

HLGT high-level group term, NEC not elsewhere classified

\section{Discussion}

This analysis has found, among reports of adverse events associated with common antimalarial drugs, evidence of a distinct neuropsychiatric syndrome class that is strongly associated with mefloquine use. This syndrome class is defined by a very high probability $(82.7 \%)$ of symptoms of deliria, including confusion and disorientation, and a moderate probability of other serious psychiatric and neurologic symptoms including dementia and amnestic conditions $(18.6 \%)$, and seizures $(18.1 \%)$. Among reports of adverse events associated with drugs used for the prophylaxis of chloroquine-resistant malaria, this syndrome class is nearly exclusive to the use of mefloquine, and occurs in approximately one in six such reports.

Intriguingly, the characteristic symptoms of this syndrome class, including confusion, amnesia, and seizure, were among the earliest reported, serious adverse neuropsychiatric effects of mefloquine noted in the literature following the drug's initial European licensing in the mid1980s. Initial case reports described a syndrome of acute brain or toxic encephalopathy, typically with confusion and seizure [26-28]. Isolated amnesia was also reported [29], as was psychosis associated with symptoms of confusion [30]. An early case series, which emphasized neurological reactions, also noted that difficulties with concentration were a common feature [31]. Unlike other possibly more subjective neuropsychiatric symptoms, these severe neuropsychiatric reactions, suggestive of organic central nervous system dysfunction, would be expected to have been subject to minimal reporting bias or nocebo effect.

In this study, among reports of adverse events, this syndrome is commonly accompanied by clinical features that are considered prodromal when associated with mefloquine use, including sleep disturbance, abnormal dreams, anxiety, and depression, as well as neurological symptoms such as dizziness, vertigo, and paresthesias [2]. While this study's methods could not determine whether any of these reported symptoms preceded the more serious characteristic symptoms of this syndrome class, such progression would be consistent with case reports [13-15] and regulatory warnings describing certain neuropsychiatric symptoms as prodromal to more serious events from the use of mefloquine. Similarly, while this study's methods could not determine the chronicity of these symptoms, their persistence would be consistent with the results of a recent cohort study of reported mefloquine adverse events, which found symptoms including mood disturbance, cognitive dysfunction, and nightmares occasionally persisting for months to years after the initial adverse event report [32].

Interestingly, this study found that this syndrome class is more common among reports of adverse events from male users than female users of mefloquine. This contrasts with other published findings, in which milder neuropsychiatric 
Table 6 Prevalence of most commonly reported HLGTs and reactions within each, by PT, among cases assigned to mostlikely syndrome class, two-class HLGT-level latent class model, by class

\begin{tabular}{|c|c|c|c|}
\hline & \multirow{2}{*}{$\begin{array}{l}\text { Syndrome } \\
n(\%)\end{array}$} & \multicolumn{2}{|c|}{ Non-syndrome } \\
\hline & & $n(\%)$ & OR $(95 \% \mathrm{CI})$ \\
\hline All cases & $237(100)$ & $5095(100)$ & \\
\hline Deliria (including confusion) & $181(76.4)$ & $16(0.3)$ & $1039.2(582.1-1855.4)$ \\
\hline Confusional state & $114(48.1)$ & $11(0.2)$ & \\
\hline Disorientation & $55(23.2)$ & $5(0.1)$ & \\
\hline Delirium & $25(10.5)$ & $0(0.0)$ & \\
\hline Neurological disorders NEC & $109(46.0)$ & $821(16.1)$ & $4.3(3.3-5.6)$ \\
\hline Dizziness & $41(17.3)$ & $234(4.6)$ & \\
\hline Hypoesthesia & $17(7.2)$ & $128(2.5)$ & \\
\hline Paresthesia & $16(6.8)$ & $93(1.8)$ & \\
\hline Vertigo & $15(6.3)$ & $52(1.0)$ & \\
\hline Anxiety disorders and symptoms & $98(41.4)$ & $412(8.1)$ & $7.7(5.8-10.2)$ \\
\hline Anxiety & $55(23.2)$ & $245(4.8)$ & \\
\hline Agitation & $34(14.3)$ & $50(1.0)$ & \\
\hline Panic attack & $26(11.0)$ & $101(2.0)$ & \\
\hline Sleep disorders and disturbances & $84(35.4)$ & $318(6.2)$ & $7.9(5.9-10.6)$ \\
\hline Insomnia & $39(16.5)$ & $147(2.9)$ & \\
\hline Somnolence & $21(8.9)$ & $47(0.9)$ & \\
\hline Abnormal dreams & $19(8.0)$ & $50(1.0)$ & \\
\hline Nightmare & $18(7.6)$ & $65(1.3)$ & \\
\hline Sleep disorder & $16(6.8)$ & $26(0.5)$ & \\
\hline Depressed mood disturbances and disorders & $74(31.2)$ & $238(4.7)$ & $8.9(6.6-12.1)$ \\
\hline Depression & $62(26.2)$ & $185(3.6)$ & \\
\hline
\end{tabular}

$C I$ confidence interval, $H L G T$ high-level group term, $N E C$ not elsewhere classified, $O R$ odds ratio, $P T$ preferred term symptoms, some of which might be considered prodromal, are more commonly reported among female users of the drug [33, 34]. While the reasons for this are unclear, it is plausible that this finding could reflect a greater propensity among certain male individuals, such as military personnel, to fail to discontinue mefloquine at the onset of prodromal symptoms [11], consequently risking the development of more serious neuropsychiatric effects [2].

\subsection{Limitations}

This study has several important limitations that require the results to be interpreted with caution. This study relied on reported drug adverse event data within the FAERS database, which is known to suffer from significantly incomplete reporting $[35,36]$, and the accuracy of these data, including suspect drug elements, is often questionable [35]. Partially to address the potential for suspect drug misattribution, this study included reports in the analysis even when the drug was listed as non-suspect. This had the benefit of significantly increasing the sample size, but possibly as a consequence, there were a high number of cases of the neuropsychiatric syndrome class associated with both doxycycline and loperamide. In contrast, in the post-hoc analysis of the study dataset, based on most-likely class assignment, there were no cases of the syndrome class among those in whom doxycycline was reported as indicated for malaria prophylaxis.

This study employed highly conservative methods for identifying residual conditional dependence in the partial model, as well as differential measurement in the SOC- and HLGT-level models. While this was done for computational simplicity, it may have had the effect of over-identification. Additionally, such methods, intended to exclude from the final LCM those HLGT-level indicators with any evidence of differential measurement by drug, essentially excluded from the syndrome class any reactions that might be uniquely associated with mefloquine use. While these methods were deemed desirable to guard against the risk that over-reporting of certain symptoms, such as from a nocebo effect with mefloquine use, would affect the validity of the results, this had the consequence that certain more serious reactions associated with mefloquine in later case reports, such as psychosis [14, 15], were not included among the characteristic features of the syndrome. Additionally, we did not specifically assess for evidence of 
differential measurement by age, sex, or drug indication, which may limit the validity of inference on these covariates.

Despite these limitations, these results are nonetheless useful and intriguing, and suggest that case finding for more serious neuropsychiatric reactions to mefloquine may be improved through the identification of characteristic reactions that are highly specific to the identified syndrome class. In this respect, it is noteworthy that 181 of the 197 cases $(91.9 \%)$ in the study dataset reporting one or more reactions within the HLGT that included the PTs confusional state, disorientation, and delirium, were assigned by the model to the most-likely syndrome class, and that this association was exceedingly strong and significant $(\mathrm{OR}=$ 1039.2, 95\% CI 581.1-1855.4, $p<0.001)$. These results suggest that among those users reporting adverse neuropsychiatric reactions to common antimalarial medications, and for whom no other likely cause, such as concussion, may be identified for these symptoms, such reactions should be considered nearly pathognomonic for this syndrome class.

\section{Conclusions}

Early reports of adverse effects from mefloquine described a clinical syndrome characterized principally by confusion, amnesia, and seizure. This analysis has found evidence of a similar syndrome class in subsequent reports of adverse events from common antimalarial drugs within the FAERS database. This syndrome class is strongly and significantly associated with mefloquine use, particularly in prophylaxis, and is not strongly associated with reports from other common antimalarial drugs when used in the prophylaxis and treatment of chloroquine-resistant malaria. The association of this specific and severe syndrome class with common symptoms that are considered prodromal, including anxiety, depression, sleep disturbance, and abnormal dreams, should reinforce existing regulatory guidance that the drug should be discontinued at their onset. The clinical identification of additional features associated with this syndrome class, including neurological symptoms such as dizziness, vertigo, and paresthesias, may aid in improving case finding in pharmacovigilance studies of more serious adverse reactions to the drug.

Acknowledgements The authors thank Dr. Peter Zandi for his assistance with various aspects of study design and interpretation, and for reviewing early drafts of this article.

\section{Compliance with Ethical Standards}

Funding No specific funding was obtained for this study.
Conflict of interest Dr. Remington Nevin has been retained as a consultant and expert witness in legal cases involving claims of antimalarial toxicity. Dr. Jeannie-Marie Leoutsakos reports no conflicts of interest.

Ethics approval Analysis of the deidentified FAERS data was deemed non-human subjects research and exempt from review by the Johns Hopkins Bloomberg School of Public Health Institutional Review Board.

Open Access This article is distributed under the terms of the Creative Commons Attribution-NonCommercial 4.0 International License (http://creativecommons.org/licenses/by-nc/4.0/), which permits any noncommercial use, distribution, and reproduction in any medium, provided you give appropriate credit to the original author(s) and the source, provide a link to the Creative Commons license, and indicate if changes were made.

\section{References}

1. World Health Organization. Central nervous system reactions related to the antimalarial drug, mefloquine. WHO/MAL/89/ 1054. 1989. Available from: http://whqlibdoc.who.int/malaria/ WHO_MAL_89.1054.pdf. Accessed 5 Sep 2016.

2. Nevin RL, Byrd AM. Neuropsychiatric adverse reactions to mefloquine: a systematic comparison of prescribing and patient safety guidance in the US, UK, Ireland, Australia, New Zealand, and Canada. Neurol Ther. 2016;5:69-83.

3. US Food and Drug Administration. FDA drug safety communication: FDA approves label changes for antimalarial drug mefloquine hydrochloride due to risk of serious psychiatric and nerve side effects. July 29, 2013. Available from: http://www.fda. gov/downloads/Drugs/DrugSafety/UCM362232.pdf. Accessed 5 Sep 2016.

4. Schlagenhauf P, Steffen R. Neuropsychiatric events and travel: do antimalarials play a role? J Travel Med. 2000;7:225-6.

5. Nevin RL. Rational risk-benefit decision-making in the setting of military mefloquine policy. J Parasitol Res. 2015;2015:260106.

6. Clift S, Grabowski P. Malaria prophylaxis and the media. Lancet. 1996;348:344.

7. Nevin RL, Croft AM. Psychiatric effects of malaria and antimalarial drugs: historical and modern perspectives. Malar J. 2016;15:332.

8. Ohrt C, Richie TL, Widjaja H, et al. Mefloquine compared with doxycycline for the prophylaxis of malaria in Indonesian soldiers: a randomized, double-blind, placebo-controlled trial. Ann Intern Med. 1997;126:963-72.

9. Overbosch D, Schilthuis H, Bienzle U, et al. Atovaquone-proguanil versus mefloquine for malaria prophylaxis in nonimmune travelers: results from a randomized, double-blind study. Clin Infect Dis. 2001;33:1015-21.

10. Wallace MR, Sharp TW, Smoak B, et al. Malaria among United States troops in Somalia. Am J Med. 1996;100:49-55.

11. Boudreau E, Schuster B, Sanchez J, et al. Tolerability of prophylactic Lariam regimens. Trop Med Parasitol. 1993;44:257-65.

12. Lobel HO, Bernard KW, Williams SL, et al. Effectiveness and tolerance of long-term malaria prophylaxis with mefloquine: need for a better dosing regimen. JAMA. 1991;265:361-4.

13. Livezey J, Oliver T, Cantilena L. Prolonged neuropsychiatric symptoms in a military service member exposed to mefloquine. Drug Saf Case Rep. 2016;3:7.

14. Nevin RL. Limbic encephalopathy and central vestibulopathy caused by mefloquine: a case report. Travel Med Infect Dis. 2012;10:144-51. 
15. Peterson AL, Seegmiller RA, Schindler LS. Severe neuropsychiatric reaction in a deployed military member after prophylactic mefloquine. Case Rep Psychiatry. 2011;2011:350417.

16. Levin A. FDA warning highlights mefloquine's mental health risks. In: Psychiatric News, September 10, 2013. Available from: http://psychnews.psychiatryonline.org/doi/10.1176/appi.pn.2013. 9a25. Accessed 5 Sep 2016.

17. Nevin RL, Ritchie E. The mefloquine intoxication syndrome: a significant potential confounder in the diagnosis and management of PTSD and other chronic deployment-related neuropsychiatric disorders. In: Ritchie EC, editor. Posttraumatic stress disorder and related disorders in Combat Veterans. Cham: Springer; 2015. p. 257-78.

18. Ritchie EC, Block J, Nevin RL. Psychiatric side effects of mefloquine: applications to forensic psychiatry. J Am Acad Psychiatry Law. 2013;41:224-35.

19. Nylund KL, Asparouhov T, Muthén BO. Deciding on the number of classes in latent class analysis and growth mixture modeling: a Monte Carlo simulation study. Struct Equ Model. 2007;14:535-69.

20. Faraone SV, Tsuang MT. Measuring diagnostic accuracy in the absence of a "gold standard". Am J Psychiatry. 1994;151:650-7.

21. Brown EG, Wood L, Wood S. The medical dictionary for regulatory activities (MedDRA). Drug Saf. 1999;20:109-17.

22. Steffen R. Travel medicine: prevention based on epidemiological data. Trans R Soc Trop Med Hyg. 1991;85:156-62.

23. Moore TJ, Cohen MR, Furberg CD. Serious adverse drug events reported to the Food and Drug Administration, 1998-2005. Arch Intern Med. 2007;167:1752-9.

24. Bandeen-Roche K, Miglioretti DL, Zeger SL, Rathouz PJ. Latent variable regression for multiple discrete outcomes. J Am Stat Assoc. 1997;92:1375-86.

25. Vermunt JK. Latent class modeling with covariates: two improved three-step approaches. Polit Anal. 2010;18:450-69.
26. Bernard J, Le Camus J, Sarrouy J, et al. Toxic encephalopathy induced by mefloquine: 3 case reports. Med Armees. 1989;17:209-11.

27. Bernard J, Le Camus J, Sarrouy J, et al. Toxic encephalopathy caused by mefloquine? Presse Med. 1987;16:1654-5.

28. Rouveix B, Bricaire F, Michon C, et al. Mefloquine and an acute brain syndrome. Ann Intern Med. 1989;110:577-8.

29. Lapras J, Vighetto A, Trillet M, Garin JP. Transient disorders of memory after a malaria attack: caused by mefloquine? Press Med. 1989;18:776.

30. Björkman A. Acute psychosis following mefloquine prophylaxis. Lancet. 1989;2:865.

31. Patchen LC, Campbell CC, Williams SB. Neurologic reactions after a therapeutic dose of mefloquine. $N$ Engl $J$ Med. 1989;321:1415-6.

32. Ringqvist $\AA$, Bech P, Glenthøj B, Petersen E. Acute and longterm psychiatric side effects of mefloquine: a follow-up on Danish adverse event reports. Travel Med Infect Dis. 2015;13:80-8.

33. van Riemsdijk MM, Sturkenboom MCJM, Ditters JM, et al. Low body mass index is associated with an increased risk of neuropsychiatric adverse events and concentration impairment in women on mefloquine. Br J Clin Pharmacol. 2004;57:506-12.

34. van Riemsdijk MM, Sturkenboom MCJM, Pepplinkhuizen L, Stricker BHC. Mefloquine increases the risk of serious psychiatric events during travel abroad: a nationwide case-control study in the Netherlands. J Clin Psychiatry. 2005;66:199-204.

35. Getz KA, Stergiopoulos S, Kaitin KI. Evaluating the completeness and accuracy of MedWatch data. Am $J$ Ther. 2012;21:442-6.

36. Ma P, Marinovic I, Karaca-Mandic P. Drug manufacturers' delayed disclosure of serious and unexpected adverse events to the US Food and Drug Administration. JAMA Intern Med. 2015;175:1565-6. 